\title{
Solution Conformations of Curcumin in DMSO
}

\author{
Cathryn A. Slabber · Craig D. Grimmer · Ross S. Robinson
}

School of Chemistry \& Physics, University of KwaZulu-Natal, Private Bag X01, Pietermaritzburg, 3209, South Africa

\begin{tabular}{lr}
\hline Item & Page \\
\hline NMR characterization of curcumin in DMSO-d6. & 2 \\
\hline Conformational search details. & 3 \\
\hline NAMFIS atom number map and NAMFIS input file. & 4 \\
\hline NAMFIS output file. & 5 \\
\hline XRD structures of curcumin (single- and multi-component) from the CSD. & 6 \\
\hline Single-component single-crystal XRD structures of curcumin, superpositions of structures. & 11 \\
Multi-component single-crystal XRD structures of curcumin, superpositions of structures. & 14 \\
\hline Interplane angles (15 atom plane including keto-enol fragment, red; 10 atom plane, green). & 16 \\
\hline Side-by-side colour and greyscale figures; enlarged Figure 5. & 21 \\
\hline
\end{tabular}




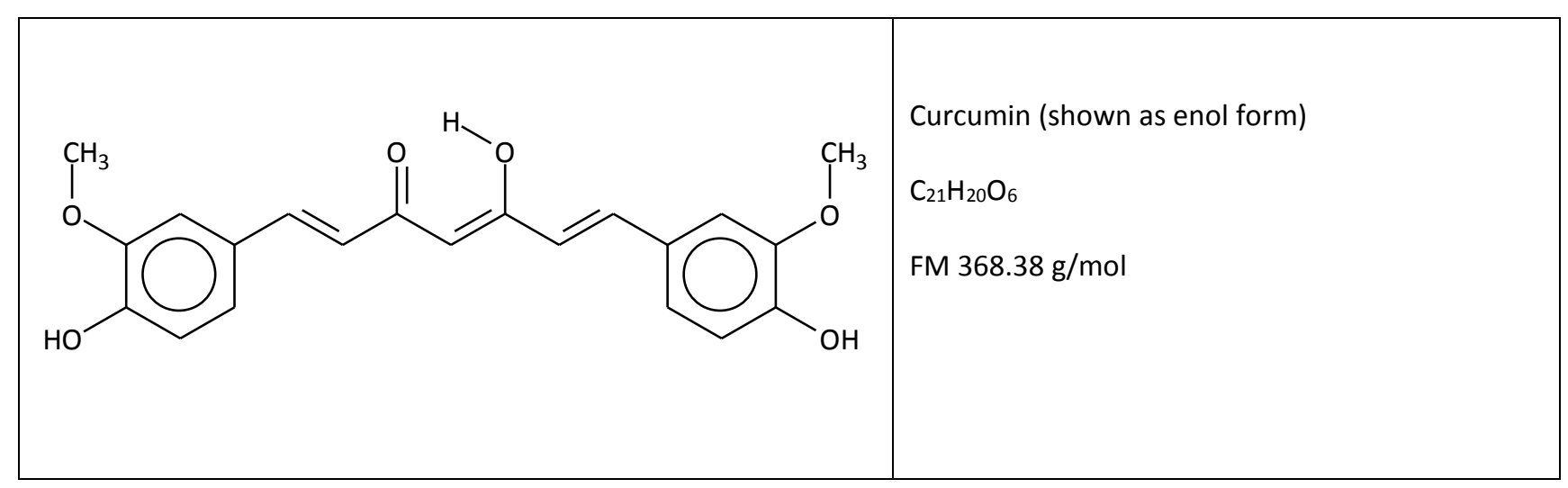

${ }^{1} \mathrm{H}$ NMR (ppm, DMSO-d 6 ): $\delta 3.84\left(\mathrm{~s}, 6 \mathrm{H}, 2 \times \mathrm{OCH}_{3}\right) ; 6.06(\mathrm{~s}, 1 \mathrm{H}, \mathrm{C}(\mathrm{O})-\mathrm{CH}-\mathrm{C}(\mathrm{OH})) ; 6.75(\mathrm{~d}, \mathrm{~J}=15.6,2 \mathrm{H}, 2 \times \mathrm{CH}=\mathrm{CH}-\mathrm{C}(\mathrm{O}))$; $6.82\left(\mathrm{~d}, J=8.2,2 \mathrm{H}, 2 \times \mathrm{HO}-\mathrm{C}_{\mathrm{q}}-\mathrm{CH}-\mathrm{CH}-\mathrm{C}_{\mathrm{q}}\right) ; 7.15\left(\mathrm{dd}, J=8.3,1.9,2 \mathrm{H}, 2 \times \mathrm{HO}-\mathrm{C}_{\mathrm{q}}-\mathrm{CH}-\mathrm{CH}-\mathrm{C}_{\mathrm{q}}\right) ; 7.32(\mathrm{~d}, J=1.8,2 \mathrm{H}$, $2 \times \mathrm{H}_{3} \mathrm{C}-\mathrm{O}-\mathrm{C}_{\mathrm{q}}-\mathrm{CH}-\mathrm{C}_{\mathrm{q}}$ ); 7.54 (d, J = 15.8, $2 \mathrm{H}, 2 \times \mathrm{CH}=\mathrm{CH}-\mathrm{C}(\mathrm{O})$ ); 9.68 (s, $2 \mathrm{H}, 2$ x phenolic $\mathrm{OH}$ ); 16.35 (s, $1 \mathrm{H}$, enolic OH).

${ }^{13} \mathrm{C}$ NMR (ppm, DMSO-d $): \delta 55.66$ (q, $\left.2 \mathrm{C}, 2 \times \mathrm{OCH}_{3}\right) ; 100.72$ (d, $\left.1 \mathrm{C}, \mathrm{C}(\mathrm{O})-\mathrm{CH}-\mathrm{C}(\mathrm{OH})\right) ; 111.35$ (d, $2 \mathrm{C}$, $2 \times \mathrm{H}_{3} \mathrm{C}-\mathrm{O}-\mathrm{C}_{\mathrm{q}}-\mathrm{CH}-\mathrm{C}_{\mathrm{q}}$ ); 115.67 (d, $\left.2 \mathrm{C}, 2 \times \mathrm{HO}-\mathrm{C}_{\mathrm{q}}-\mathrm{CH}-\mathrm{CH}-\mathrm{C}_{\mathrm{q}}\right) ; 121.05$ (d, $\left.2 \mathrm{C}, 2 \times \mathrm{CH}=\mathrm{CH}-\mathrm{C}(\mathrm{O})\right) ; 123.05$ (d, $2 \mathrm{C}$, $2 \times \mathrm{HO}_{-} \mathrm{C}_{\mathrm{q}}-\mathrm{CH}-\mathrm{CH}-\mathrm{C}_{\mathrm{q}}$ ); 126.28 (s, $2 \mathrm{C}, 2 \times \mathrm{C}_{\mathrm{q}}-\mathrm{CH}=\mathrm{CH}$ ); 140.63 (d, $2 \mathrm{C}, 2 \times \mathrm{CH}=\mathrm{CH}-\mathrm{C}(\mathrm{O})$ ); 147.95 (s, $2 \mathrm{C}, 2 \times \mathrm{C}_{\mathrm{q}}-\mathrm{OCH}$ ); 149.33 (s, 2 C, $\left.2 \times \mathrm{C}_{\mathrm{q}}-\mathrm{OH}\right) ; 183.14$ (s, $\left.2 \mathrm{C}, 2 \times \mathrm{C}(\mathrm{O})-\mathrm{CH}-\mathrm{C}(\mathrm{OH})\right)$.

\begin{tabular}{|c|c|}
\hline${ }^{1} \mathrm{H}$ NMR (ppm, DMSO-d $)$ & ${ }^{13} \mathrm{C}$ NMR (ppm, DMSO-d 6 ) \\
\hline $3.84\left(\mathrm{~s}, 6 \mathrm{H}, 2 \times \mathrm{OCH}_{3}\right)$ & $55.66\left(q, 2 \mathrm{C}, 2 \times \mathrm{OCH}_{3}\right)$ \\
\hline $6.06(\mathrm{~s}, 1 \mathrm{H}, \mathrm{C}(\mathrm{O})-\mathrm{CH}-\mathrm{C}(\mathrm{OH}))$ & $100.72(\mathrm{~d}, 1 \mathrm{C}, \mathrm{C}(\mathrm{O})-\mathrm{CH}-\mathrm{C}(\mathrm{OH}))$ \\
\hline $6.75(\mathrm{~d}, \mathrm{~J}=15.6,2 \mathrm{H}, 2 \times \mathrm{CH}=\mathrm{CH}-\mathrm{C}(\mathrm{O}))$ & 121.05 (d, 2 C, $2 \times \mathrm{CH}=\mathrm{CH}-\mathrm{C}(\mathrm{O}))$ \\
\hline $6.82\left(\mathrm{~d}, J=8.2,2 \mathrm{H}, 2 \times \mathrm{HO}-\mathrm{C}_{\mathrm{q}}-\mathrm{CH}-\mathrm{CH}-\mathrm{C}_{\mathrm{q}}\right)$ & $115.67\left(\mathrm{~d}, 2 \mathrm{C}, 2 \times \mathrm{HO}-\mathrm{C}_{\mathrm{q}}-\mathrm{CH}-\mathrm{CH}-\mathrm{C}_{q}\right)$ \\
\hline $7.15\left(\mathrm{dd}, J=8.3,1.9,2 \mathrm{H}, 2 \times \mathrm{HO}-\mathrm{C}_{\mathrm{q}}-\mathrm{CH}-\mathrm{CH}-\mathrm{C}_{\mathrm{q}}\right)$ & $123.05\left(\mathrm{~d}, 2 \mathrm{C}, 2 \times \mathrm{HO}-\mathrm{C}_{\mathrm{q}}-\mathrm{CH}-\mathrm{CH}-\mathrm{C}_{q}\right)$ \\
\hline $7.32\left(\mathrm{~d}, J=1.8,2 \mathrm{H}, 2 \times \mathrm{H}_{3} \mathrm{C}-\mathrm{O}-\mathrm{C}_{\mathrm{q}}-\mathrm{CH}-\mathrm{C}_{\mathrm{q}}\right)$ & $111.35\left(\mathrm{~d}, 2 \mathrm{C}, 2 \times \mathrm{H}_{3} \mathrm{C}-\mathrm{O}-\mathrm{C}_{\mathrm{q}}-\mathrm{CH}-\mathrm{C}_{\mathrm{q}}\right)$ \\
\hline $7.54(\mathrm{~d}, \mathrm{~J}=15.8,2 \mathrm{H}, 2 \times \mathrm{CH}=\mathrm{CH}-\mathrm{C}(\mathrm{O}))$ & $140.63(\mathrm{~d}, 2 \mathrm{C}, 2 \times \mathrm{CH}=\mathrm{CH}-\mathrm{C}(\mathrm{O}))$ \\
\hline \multicolumn{2}{|l|}{$9.68(\mathrm{~s}, 2 \mathrm{H}, 2 \times$ phenolic $\mathrm{OH})$} \\
\hline \multicolumn{2}{|l|}{16.35 (s, $1 \mathrm{H}$, enolic $\mathrm{OH})$} \\
\hline & $126.28\left(\mathrm{~s}, 2 \mathrm{C}, 2 \times \mathrm{C}_{\mathrm{q}}-\mathrm{CH}=\mathrm{CH}\right)$ \\
\hline & $147.95\left(\mathrm{~s}, 2 \mathrm{C}, 2 \times \mathrm{C}_{\mathrm{q}}-\mathrm{OCH}_{3}\right)$ \\
\hline & $149.33\left(\mathrm{~s}, 2 \mathrm{C}, 2 \times \mathrm{C}_{\mathrm{q}}-\mathrm{OH}\right)$ \\
\hline & $183.14(\mathrm{~s}, 2 \mathrm{C}, 2 \times \mathrm{C}(\mathrm{O})-\mathrm{CH}-\mathrm{C}(\mathrm{OH}))$ \\
\hline
\end{tabular}

The analyte sample was prepared by dissolving curcumin (10 mg; Sigma-Aldrich) in DMSO-d 6 (600 $\mu$; Merck). NMR experiments were performed at the School of Chemistry and Physics (Pietermaritzburg), on a Bruker Avance-III 500 spectrometer equipped with a $5 \mathrm{~mm} \mathrm{BBOZ}\left({ }^{31} \mathrm{P}-{ }^{109} \mathrm{Ag} /{ }^{1} \mathrm{H}\right)$ probe operating at $500 \mathrm{MHz}\left({ }^{1} \mathrm{H}\right)$ and $125 \mathrm{MHz}\left({ }^{13} \mathrm{C}\right)$ with Topspin 2.1 (patch level 6) acquisition software. Experiments were performed with temperature regulation at $30{ }^{\circ} \mathrm{C}$. All data were processed with Topspin 2.1 (patch level 6). ${ }^{1}{ }^{1} \mathrm{H} 90^{\circ}$ pulse calibration was conducted using the analyte sample, and a ${ }^{13} \mathrm{C} 90^{\circ}$ pulse calibration was conducted with a $40 \%$-dioxane : $60 \%$ benzene-d 6 sample. Standard Bruker pulse programs were used for ${ }^{1} \mathrm{H},{ }^{13} \mathrm{C}-\left\{{ }^{1} \mathrm{H}\right\}$, gCOSY, DEPT, gHSQC, gHMBC, and gNOESY experiments. Internuclear distances were calculated from integrated NOE cross-peak volumes at a mixing time of $180 \mathrm{~ms}$ using an isolated-spin-pair-approximation (ISPA). A reference distance of $2.50 \AA$ between ortho-positioned protons on an aromatic ring (Figure 5) was used for the calculations. 
Conformational search details (keto form).

\begin{tabular}{|l|l||l|l|l|l|l|}
\hline & $\mathrm{MM3}$ & $\mathrm{MM3}$ & $\mathrm{MMFF}$ & $\mathrm{MMFF}$ & OPLS-2005 & OPLS-2005 \\
\hline & $\mathrm{CHCl}_{3}$ & $\mathrm{H}_{2} \mathrm{O}$ & $\mathrm{CHCl}_{3}$ & $\mathrm{H}_{2} \mathrm{O}$ & $\mathrm{CHCl}_{3}$ & $\mathrm{H}_{2} \mathrm{O}$ \\
\hline $\begin{array}{l}\# \\
\text { conformers }\end{array}$ & 10000 & 10000 & 10000 & 10000 & 10000 & 10000 \\
\hline$\#$ unique & 91 & 297 & 427 & 403 & 521 & 658 \\
\hline $\begin{array}{l}\text { Global } \\
\text { energy } \\
\text { minimum } \\
\text { (kJ/mol) / } \\
\text { (kcal/mol) }\end{array}$ & 3.15 & 41.58 & 102.69 & 94.82 & -124.64 & -143.47 \\
\hline $\begin{array}{l}\text { \#global } \\
\text { min }\end{array}$ & 1 & 1 & 1 & 1 & 3 & 1 \\
\hline
\end{tabular}

Conformational search details (enol form).

\begin{tabular}{|c|c|c|c|c|c|c|}
\hline & MM3 & MM3 & MMFF & MMFF & OPLS-2005 & OPLS-2005 \\
\hline & $\mathrm{CHCl}_{3}$ & $\mathrm{H}_{2} \mathrm{O}$ & $\mathrm{CHCl}_{3}$ & $\mathrm{H}_{2} \mathrm{O}$ & $\mathrm{CHCl}_{3}$ & $\mathrm{H}_{2} \mathrm{O}$ \\
\hline $\begin{array}{l}\text { \# } \\
\text { conformers }\end{array}$ & 10000 & 10000 & 10000 & 10000 & 10000 & 10000 \\
\hline \# unique & 286 & 260 & 288 & 219 & 357 & 362 \\
\hline $\begin{array}{l}\text { Global } \\
\text { energy } \\
\text { minimum } \\
(\mathrm{kJ} / \mathrm{mol}) / \\
(\mathrm{kcal} / \mathrm{mol})\end{array}$ & -17.82 & 31.57 & 68.39 & 71.67 & -76.72 & -88.93 \\
\hline $\begin{array}{l}\text { \#global } \\
\text { min }\end{array}$ & 1 & 1 & 2 & 1 & 11 & 5 \\
\hline
\end{tabular}

The conformational searches were performed using Schrodinger's MacroModel software with applicable force fields (MM3, MMFF, OPLS-2005) in solvents chloroform and water using the Generalized-Born-Surface-Area (GBSA) constant dielectric model within an energy window of $21.0 \mathrm{~kJ} / \mathrm{mol}(5.0 \mathrm{kcal} / \mathrm{mol})$. Minimization of these conformers and subsequent elimination of duplicates resulted in a conformer pool of 1968 structures. Minimization was performed with full-matrix-Newton-Raphson (FMNR) minimization (OPLS-2005, $\mathrm{H}_{2} \mathrm{O}$ ) within the same energy window (21 kJ/mol) and elimination was based on a comparison of "heavy atoms plus $\mathrm{OH}$ and SH". Additional conformers from the Protein Data Bank (4PME, 4PMF) and from the Cambridge Structural Database (BINMEQ, BINMEQ04, BINMEQ06) were added to the conformers obtained from MacroModel.

Reference for 4PME, 4PMF: L. Ciccone, L. Tepshi, S. Nencetti, E. A. Stura, New Biotechnology, 2015, 32, (1), 54 - 64. Reference for BINMEQ: H. H. Tonnesen, J. Karlsen, A. Mostad, Acta Chemica Scandinavica B, 1982, 36, 475 - 479. Reference for BINMEQ04: F. R. Fronczek, Private Communication to CSD, 2008.

Reference for BINMEQ06: P. Sanphui, N. R. Goud, U. B. R. Khandavilli, S. Bhanoth, A. Nangia, Chemical Communications, 2011, 47, $5013-5015$. 
NAMFIS atom number map.

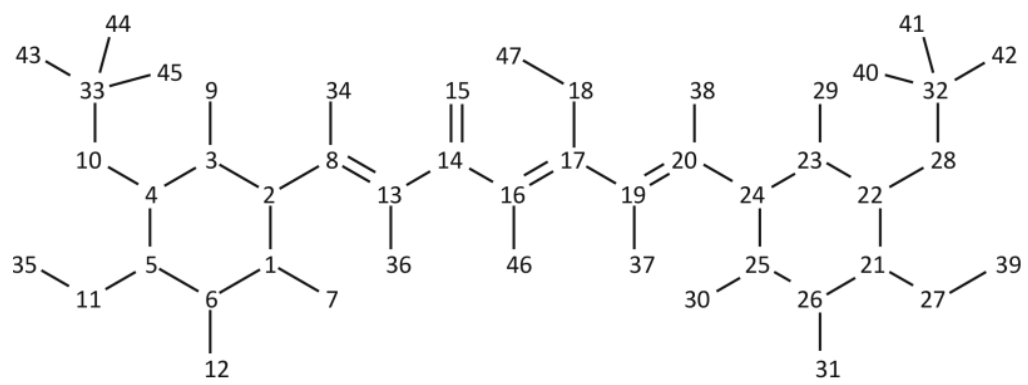

NAMFIS input file.

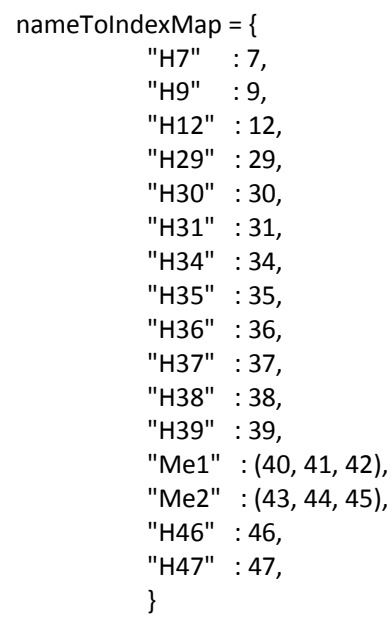

permutations $=[$

("Me1", "Me2")

("H36", "H37"),

("H34", "H38"),

("H9", "H29"),

("H7", "H30")

("H12", "H31"),

("H35", "H39"),

]

noeDistances $=[$

\{"groups" : ("H9", "Me2"), "distance" : 3.51\},

\{"groups" : ("H34", "H46"), "distance" : 2.97\},

\{"groups" : ("H36", "H46"), "distance" : 2.35\},

\{"groups" : ("H9", "H36"), "distance" : 2.58\},

\{"groups" : ("H7", "H12"), "distance" : 2.50\},

\{"groups" : ("H34", "H7"), "distance" : 2.62\},

\{"groups" : ("H34", "H9"), "distance" : 3.07\},

] 
NAMFIS output file.

\begin{tabular}{|c|c|c|c|c|c|c|}
\hline \multicolumn{2}{|c|}{ conformer } & \multicolumn{5}{|c|}{ population (\%) } \\
\hline \multicolumn{2}{|c|}{1972} & 46.64 & & & & \\
\hline \multicolumn{2}{|l|}{1243} & 40.23 & & & & \\
\hline \multicolumn{2}{|l|}{1845} & 7.46 & & & & \\
\hline \multicolumn{2}{|l|}{1973} & 5.66 & & & & \\
\hline \multicolumn{7}{|c|}{ total: 99.99} \\
\hline \multicolumn{7}{|c|}{ SSD: 2} \\
\hline \multicolumn{7}{|c|}{$1972,1243,1845,1973$} \\
\hline \multicolumn{2}{|c|}{ NOEs } & weight & experimental & calc & & difference \\
\hline H9 & Me2 & 1.00 & 3.51 & 2.88 & 0.63 & \\
\hline H34 & $\mathrm{H} 46$ & 1.00 & 2.97 & 2.98 & 0.01 & \\
\hline H36 & $\mathrm{H} 46$ & 1.00 & 2.35 & 2.42 & 0.07 & \\
\hline H9 & H36 & 1.00 & 2.58 & 2.51 & 0.07 & \\
\hline $\mathrm{H} 7$ & $\mathrm{H} 12$ & 1.00 & 2.50 & 2.47 & 0.03 & \\
\hline H34 & $\mathrm{H} 7$ & 1.00 & 2.62 & 2.69 & 0.07 & \\
\hline H34 & $\mathrm{H9}$ & 1.00 & 3.07 & 3.06 & 0.01 & \\
\hline
\end{tabular}


XRD structures of curcumin (single- and multi-component) from the CSD (hydrogen atoms omitted)

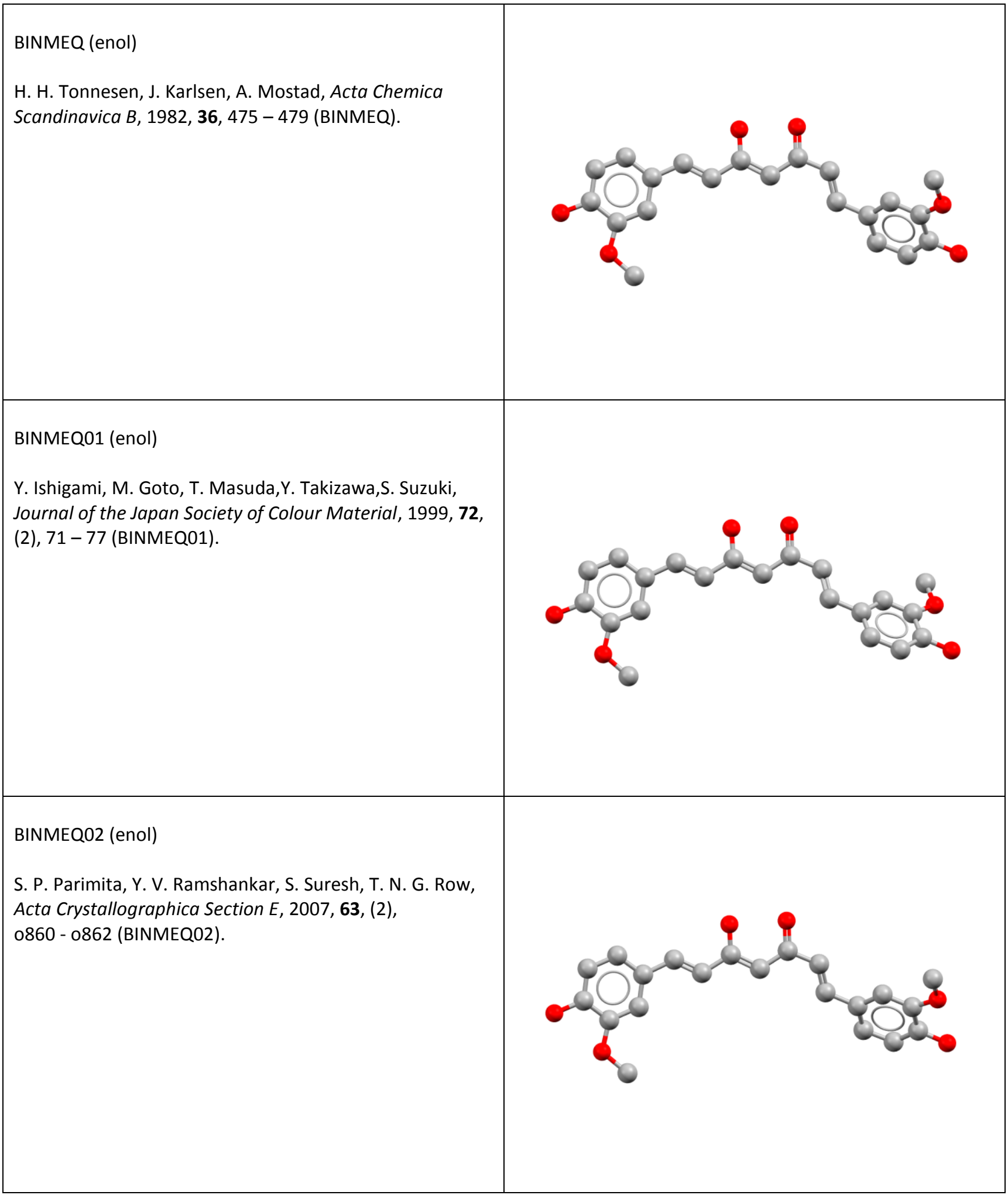




\section{BINMEQ03 (enol)}

Q-L. Suo, Y-C Huang, L-H. Weng, W-Z. He, C-P. Li, Y-X. Li, H-L. Hong, Food Science, 2006, 27, (3), $27-30$ (BINMEQ03).

BINMEQ04 (enol)

F. R. Fronczek, Private Communication to CSD, 2008 (BINMEQ04).

BINMEQ05 (enol)

P. Sanphui, N. R. Goud, U. B. R. Khandavilli, S. Bhanoth, A. Nangia, Chemical Communications, 2011, 47, 5013 5015 (BINMEQ05). 


\section{BINMEQ06 (enol)}

P. Sanphui, N. R. Goud, U. B. R. Khandavilli, S. Bhanoth, A. Nangia, Chemical Communications, 2011, 47, 5013 5015 (BINMEQ05).

BINMEQ07 (enol)

P. Sanphui, N. R. Goud, U. B. R. Khandavilli, S. Bhanoth,

A. Nangia, Chemical Communications, 2011, 47, 5013 5015 (BINMEQ07).

BINMEQ08 (enol)

A. R. Parameswari, B. Devipriya, S. J. Jenniefer,

P. T. Muthiah, P. Kumaradhas, Journal of Chemical Crystallography, 2012, 42, 227 - 231 (BINMEQ08). 
AXOGIE (enol; multi-component)

P. Sanphui, N. R. Goud, U. B. R. Khandavilli, A. Nangia, Cryst. Growth Des., 2011, 11, (9), 4135-4145.

AXOGOK (enol; multi-component)

P. Sanphui, N. R. Goud, U. B. R. Khandavilli, A. Nangia, Cryst. Growth Des., 2011, 11, (9), 4135-4145.

OJIWOV (enol; multi-component)

T. De Silva, I. M. Warner, F. R. Fronczek, Private Communication to CSD, 2016. 
QUMDEJ (enol; multi-component)

H. Su, H. He, Y. Tian, N. Zhao, F. Sun, X. Zhang, Q. Jiang, G. Zhu, Inorg. Chem. Commun., 2015, 55, 92-95.

QUMDIN (keto; multi-component)

H. Su, H. He, Y. Tian, N. Zhao, F. Sun, X. Zhang, Q. Jiang, G. Zhu, Inorg. Chem. Commun., 2015, 55, 92-95. 
Single-component single-crystal XRD structures of curcumin, superpositions of structures

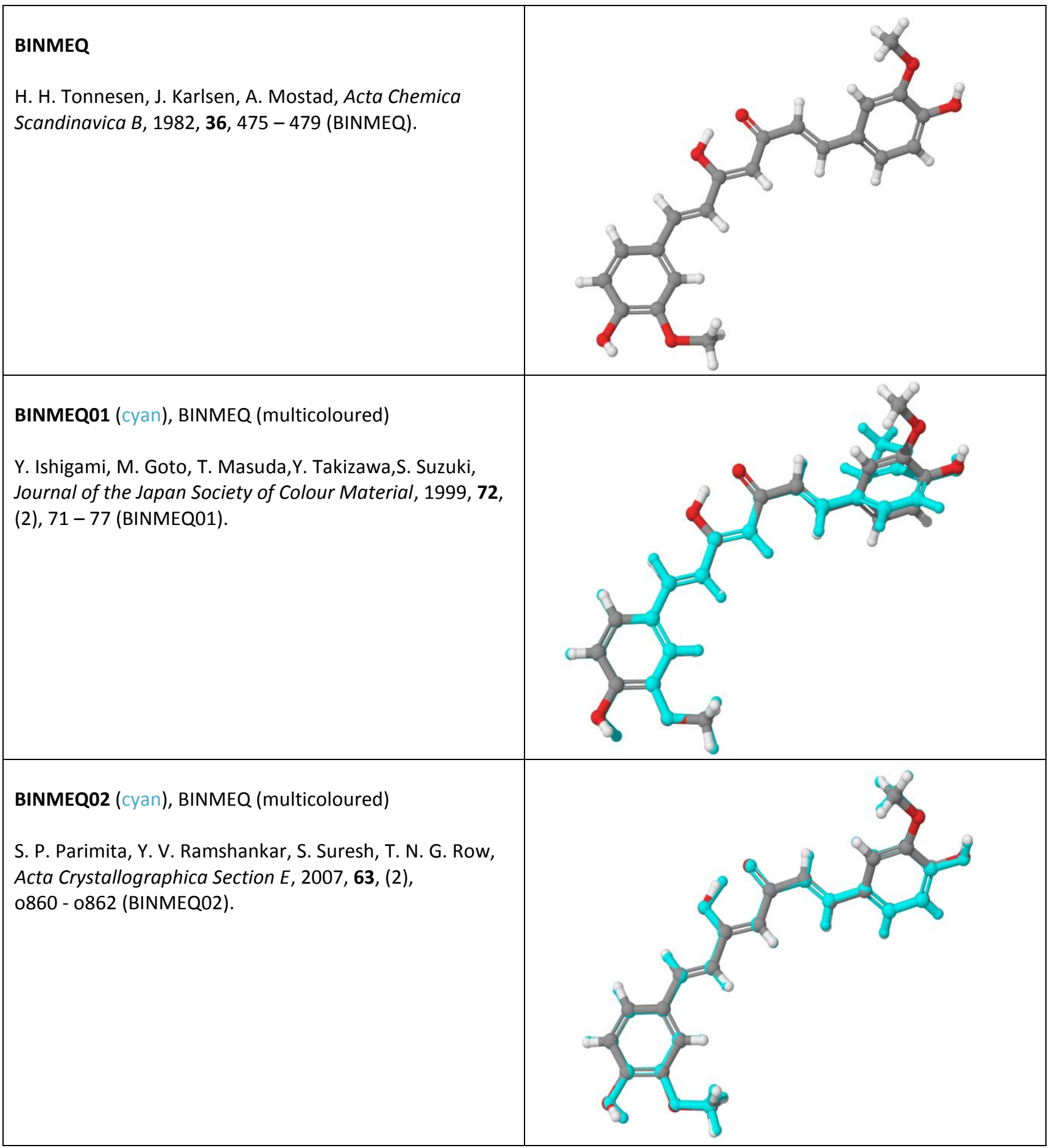


BINMEQ03 (cyan), BINMEQ (multicoloured)

Q-L. Suo, Y-C Huang, L-H. Weng, W-Z. He, C-P. Li, Y-X. Li, H-L. Hong, Food Science, 2006, 27, (3), $27-30$ (BINMEQ03).

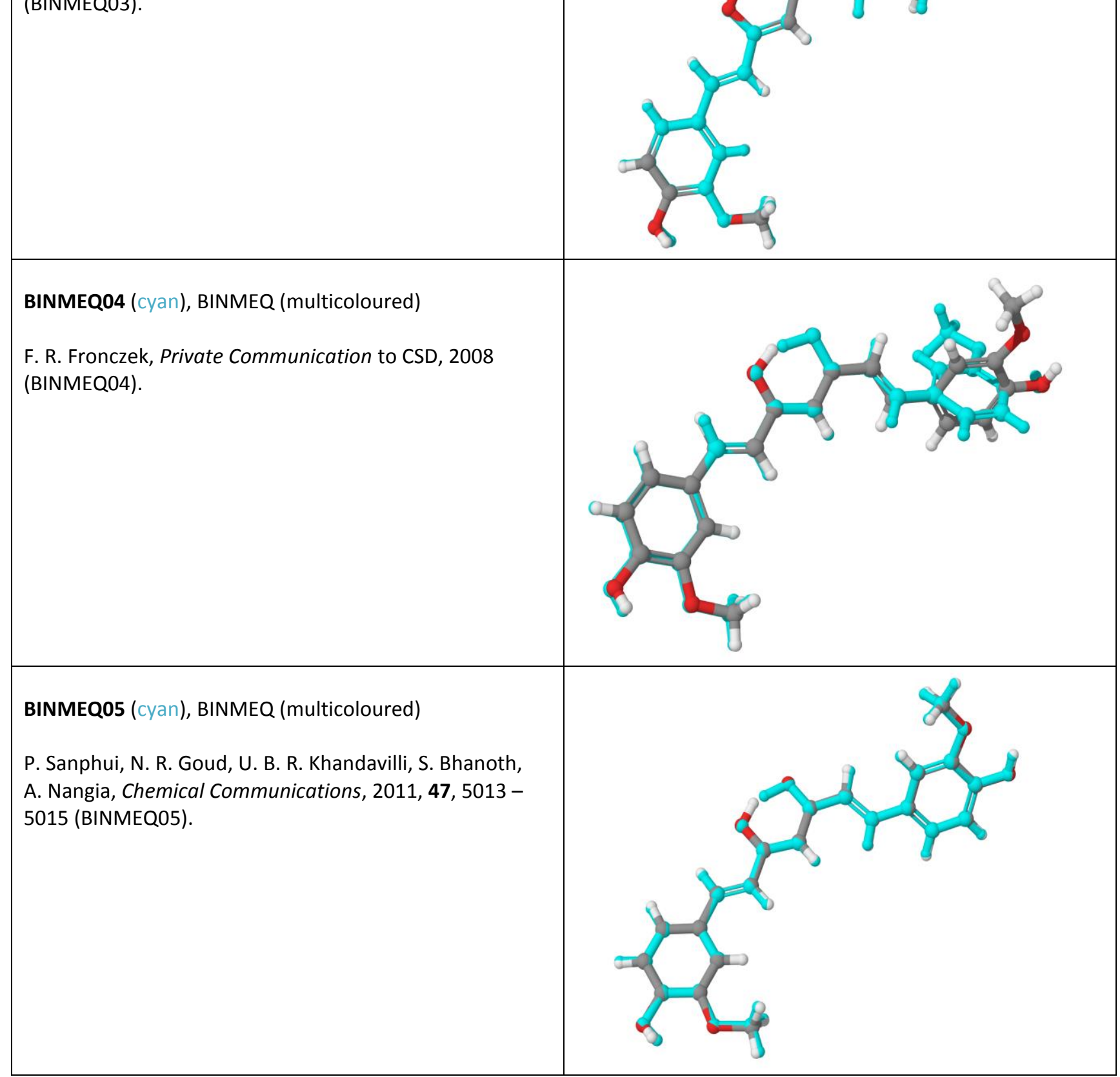




\section{BINMEQ06}

P. Sanphui, N. R. Goud, U. B. R. Khandavilli, S. Bhanoth, A. Nangia, Chemical Communications, 2011, 47, 5013 5015 (BINMEQ06).

BINMEQ07 (cyan), BINMEQ06 (multicoloured)

P. Sanphui, N. R. Goud, U. B. R. Khandavilli, S. Bhanoth, A. Nangia, Chemical Communications, 2011, 47, 5013 5015 (BINMEQ07).

BINNMEQ08 (cyan), BINMEQ06 (multicoloured)

A. R. Parameswari, B. Devipriya, S. J. Jenniefer,

P. T. Muthiah, P. Kumaradhas, Journal of Chemical Crystallography, 2012, 42, 227 - 231 (BINMEQ08).

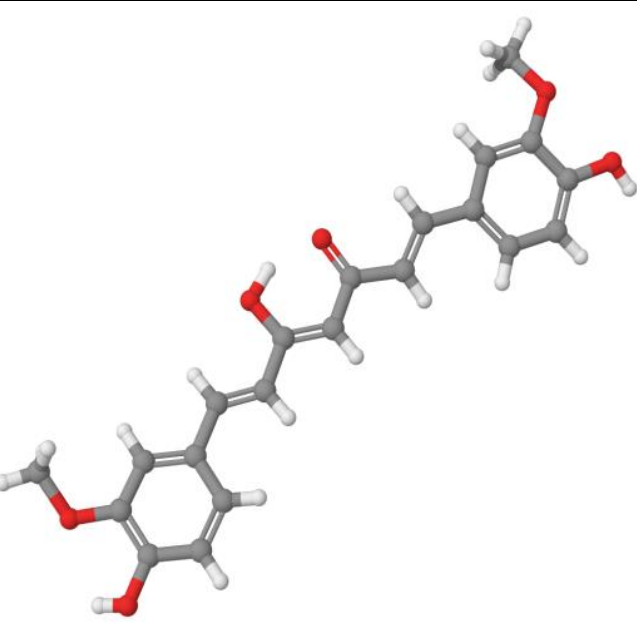


Multi-component single-crystal XRD structures of curcumin, superpositions of structures

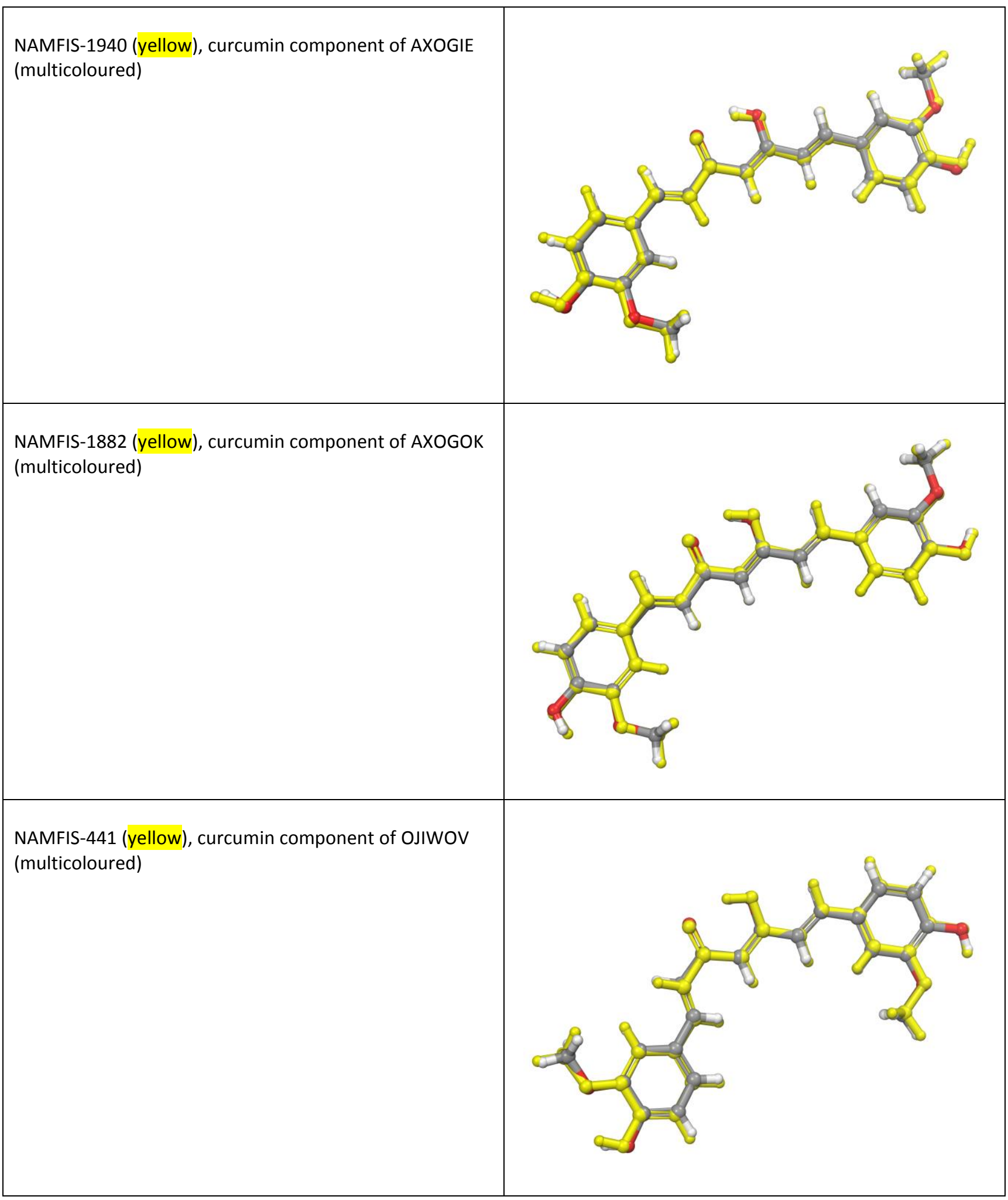




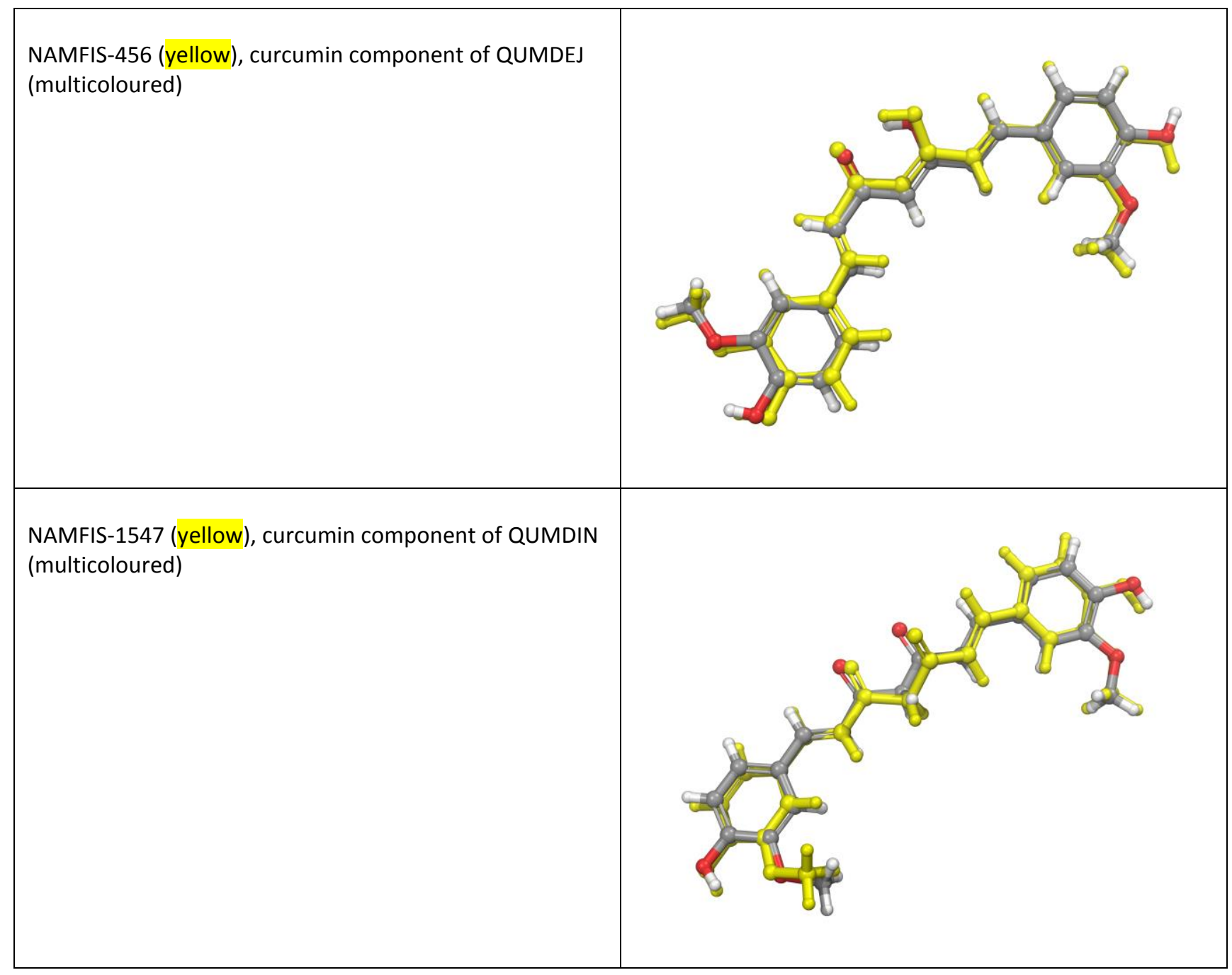


Interplane angles (15 atom plane including keto-enol fragment, red; 10 atom plane, green)

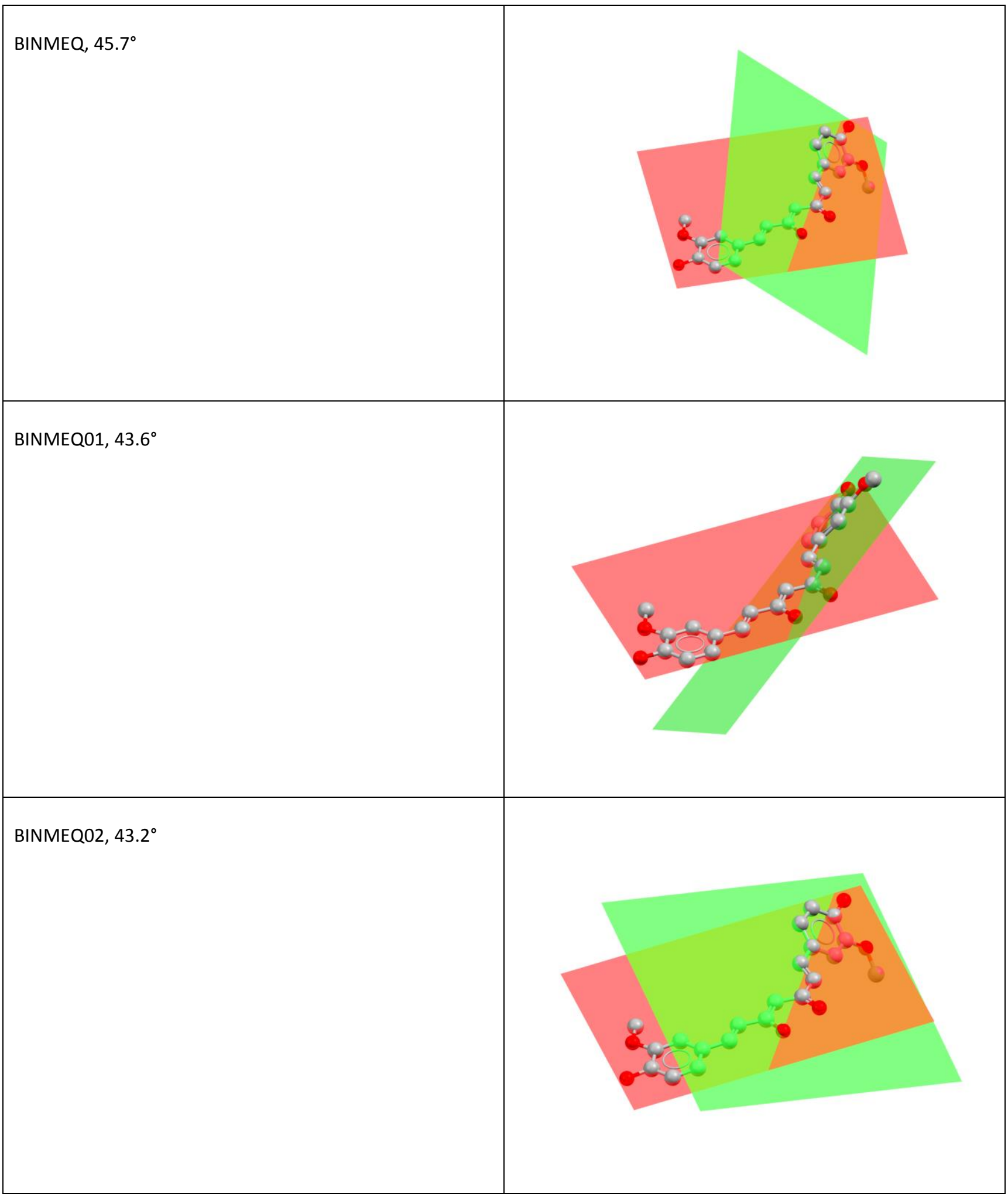




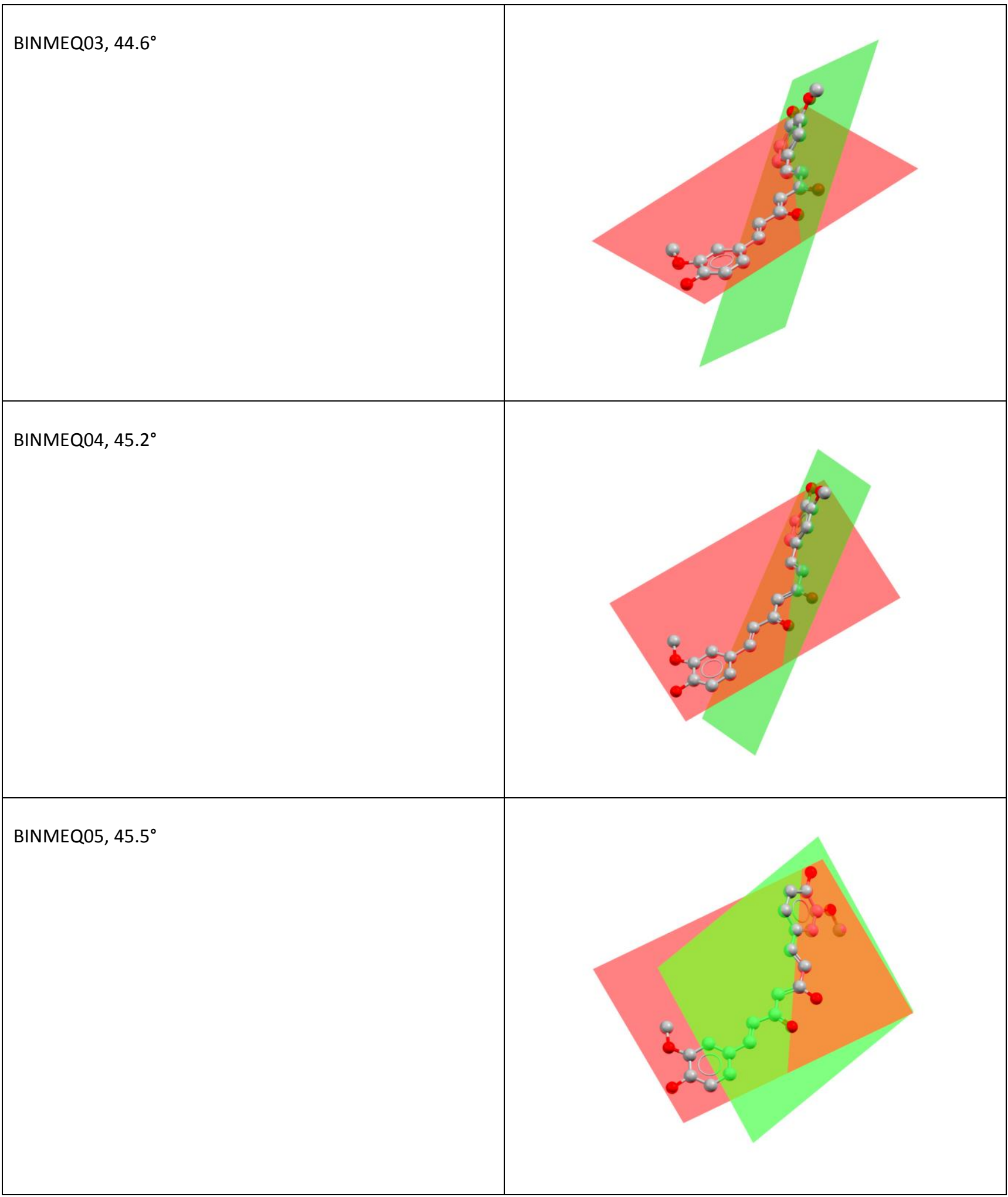




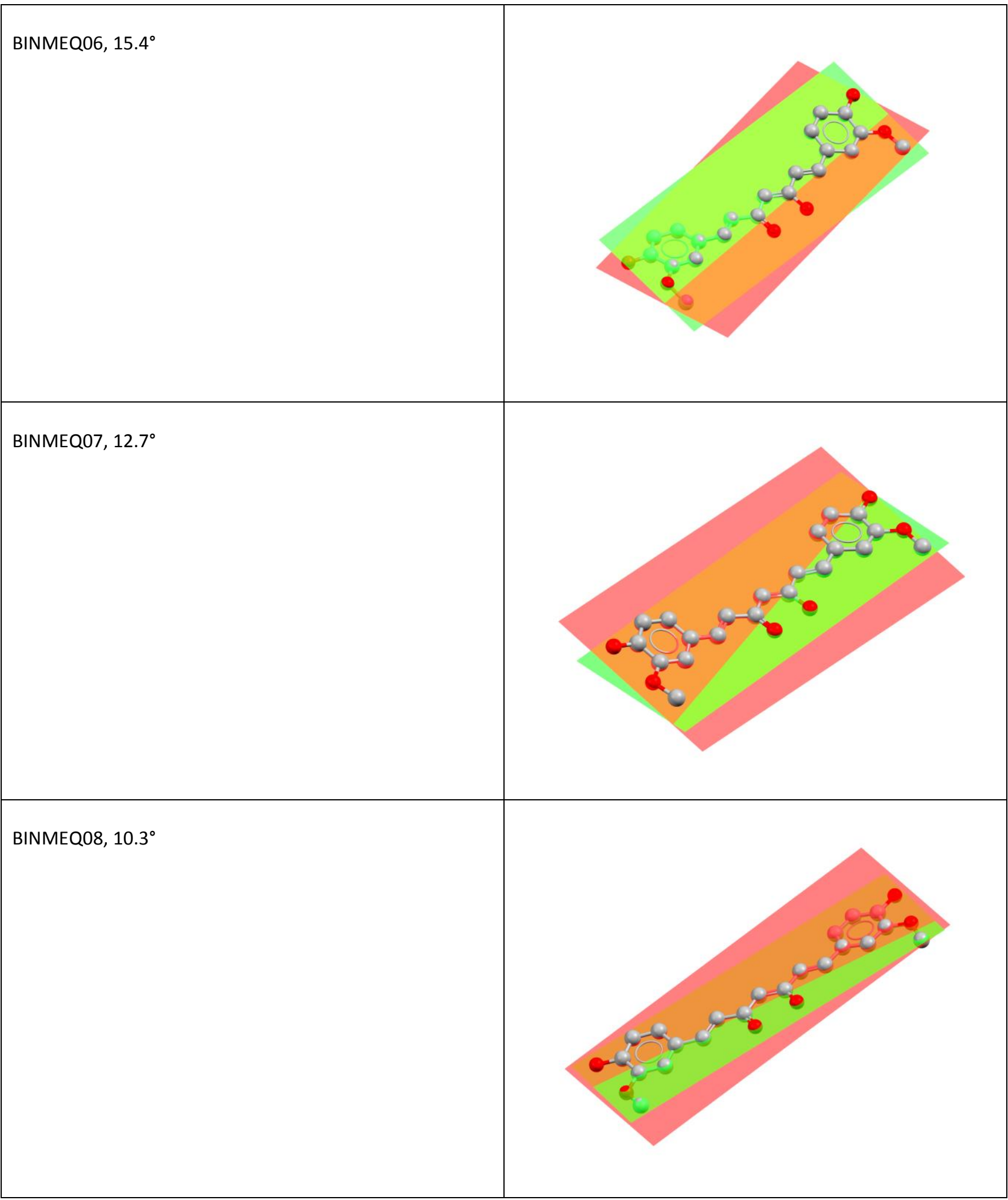




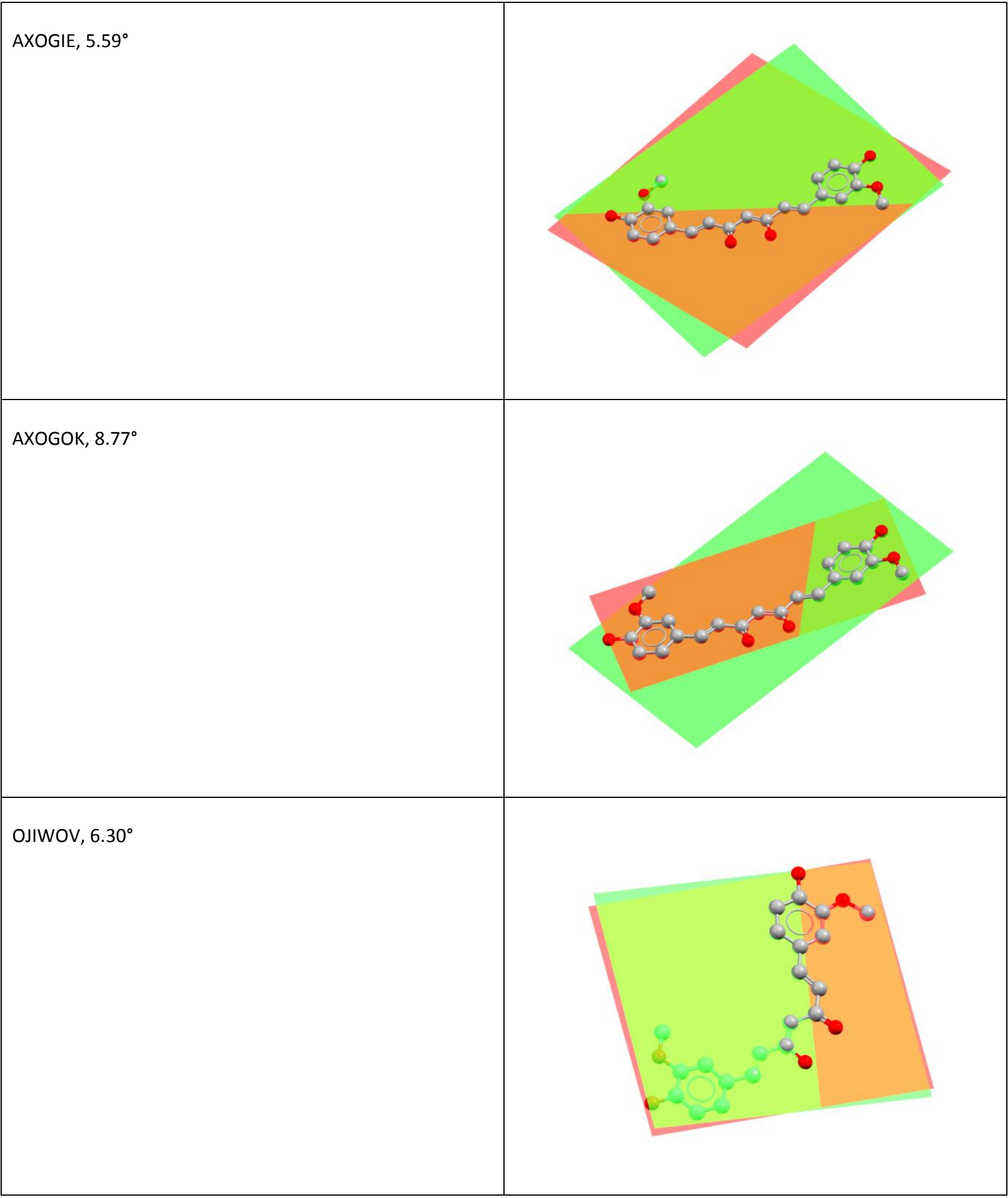




\section{QUMDEJ, $4.73^{\circ}$}

QUMDIN, $8.14^{\circ}$ 
Figure 1: Enol and keto forms, tautomerism of curcumin.

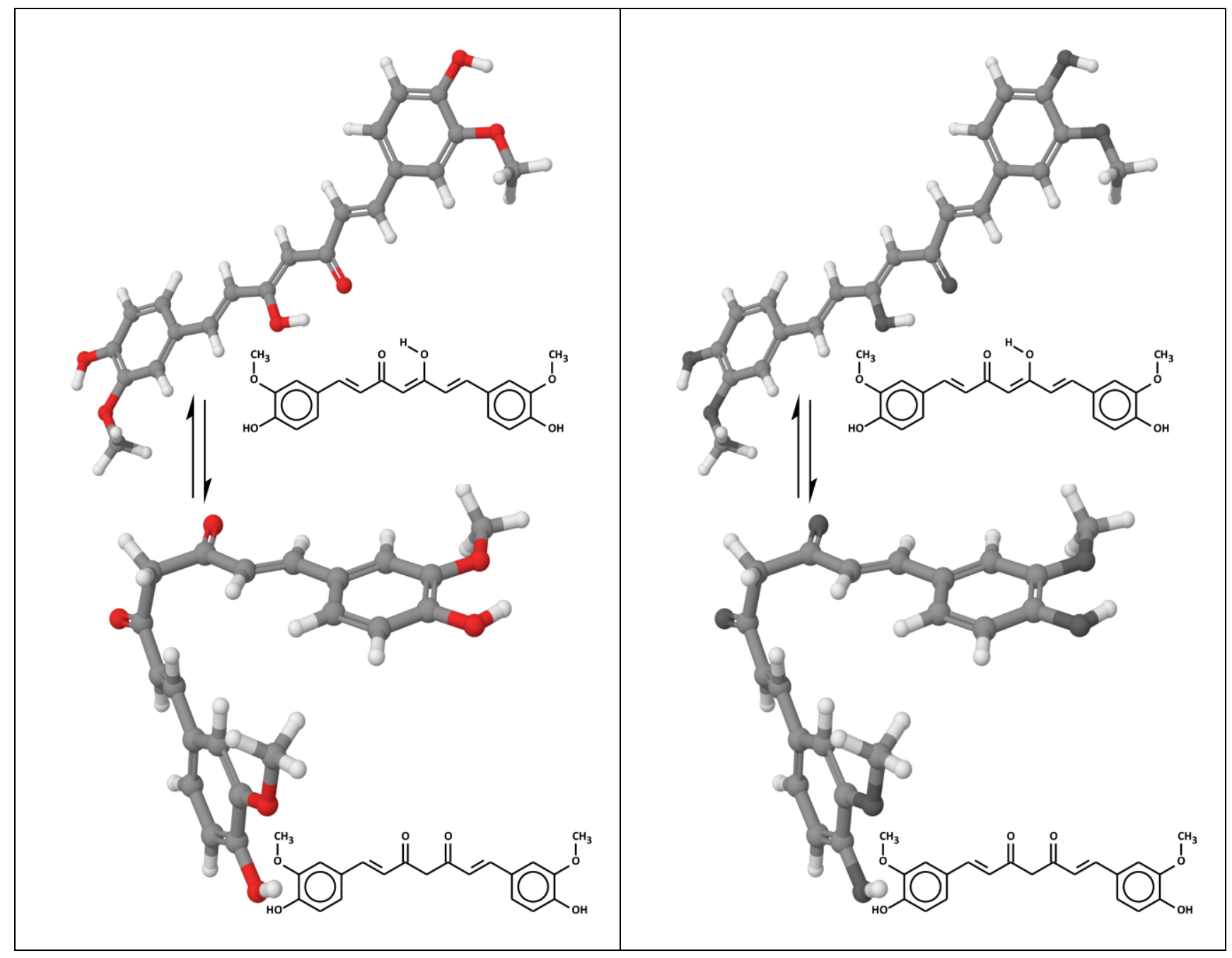

Figure 2: 4PME (ribbon) with curcumin (red; hydrogen atoms omitted for clarity).

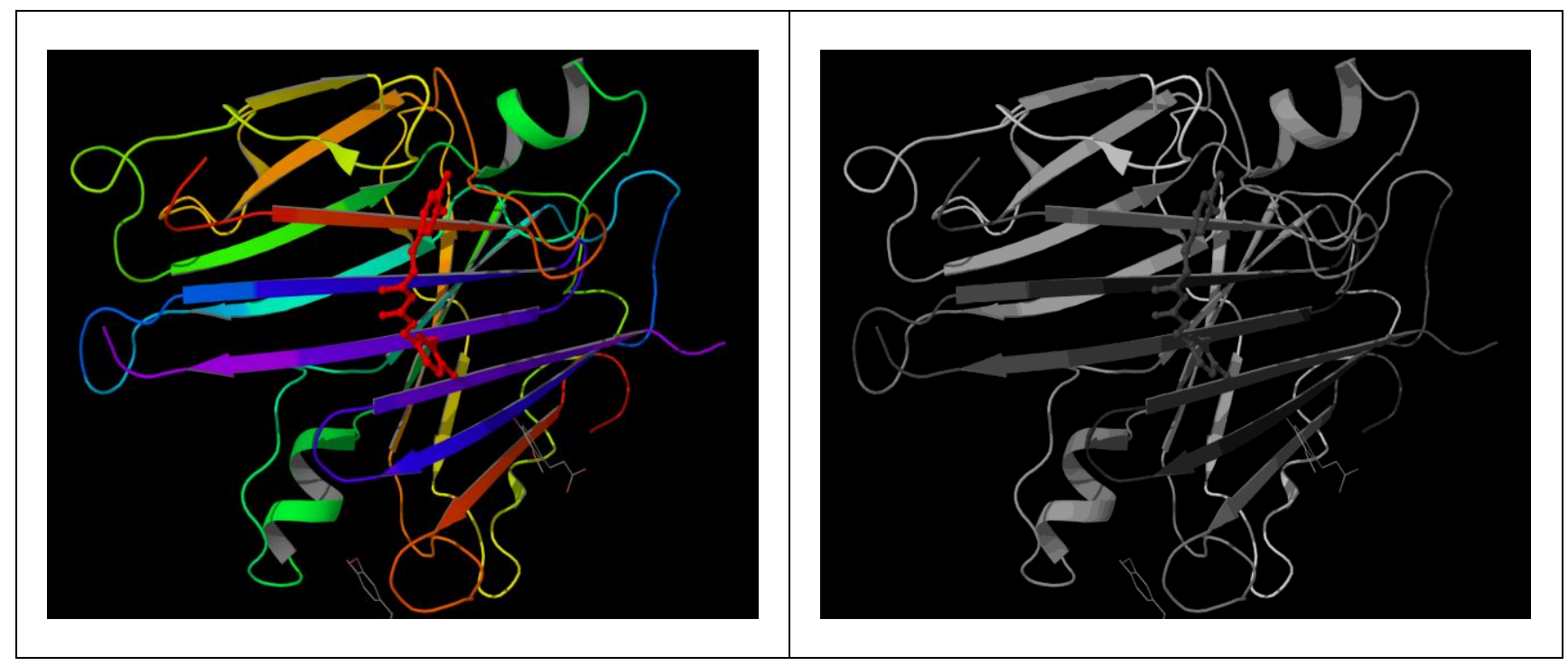


Figure S3: 4PMF (ribbon) with curcumin (red; hydrogen atoms omitted for clarity).

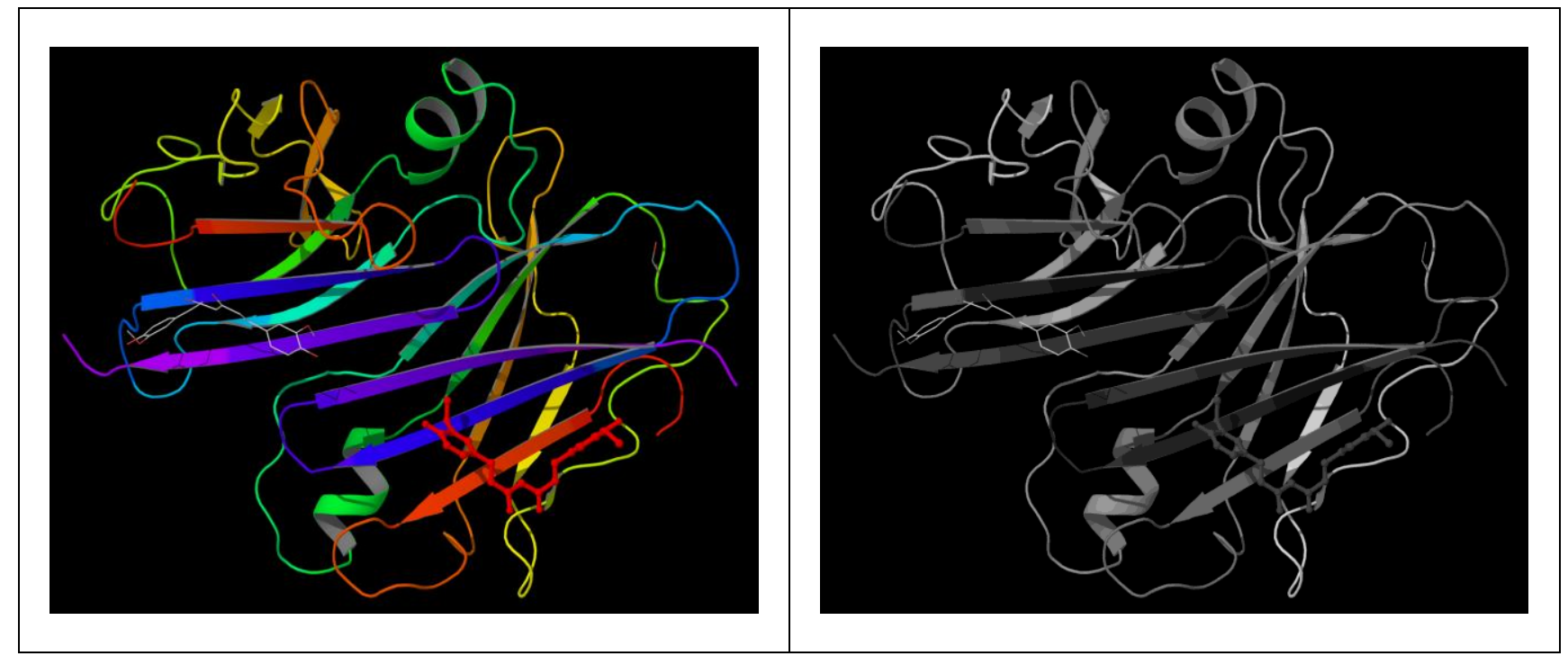

Figure 3: Superimposed E,E-curcumin molecules from 4PME (yellow, light) and 4PMF (magenta, dark); RMSD $2.81 \AA$.

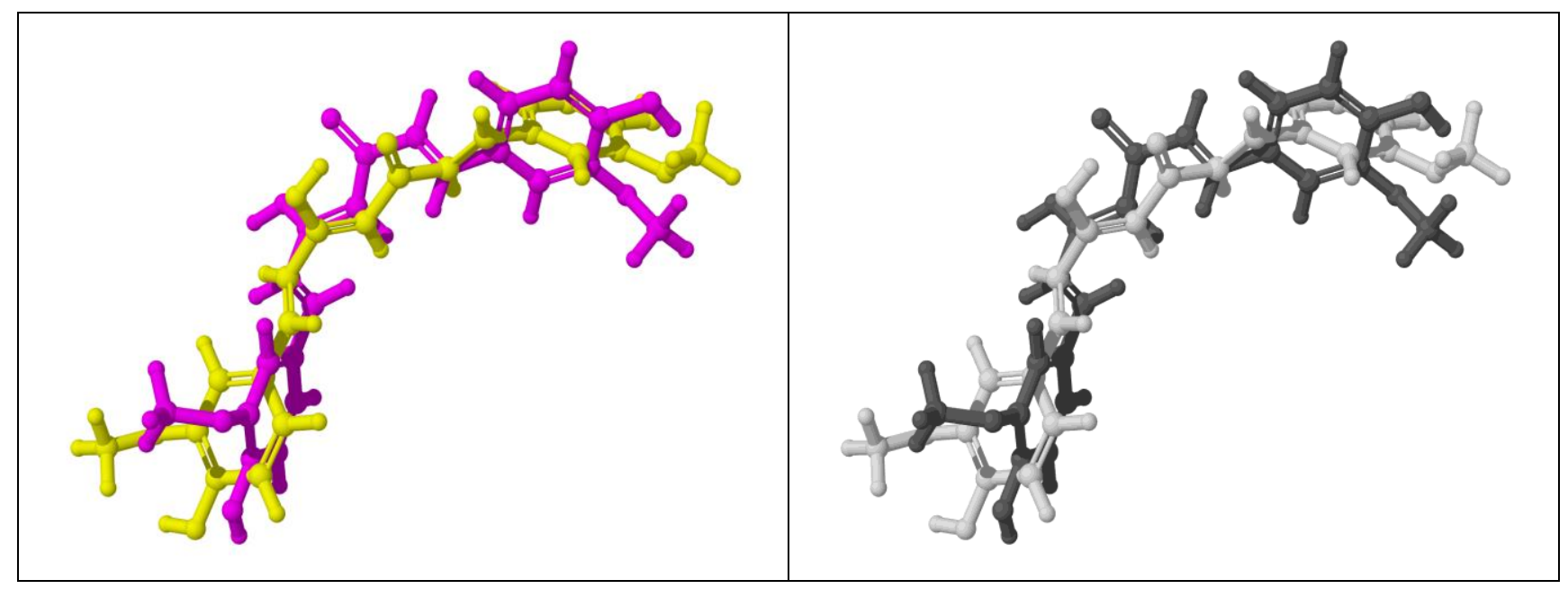




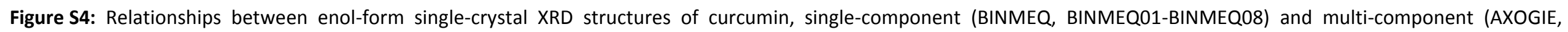
AXOGOK, OJIWOV, QUMDEJ).

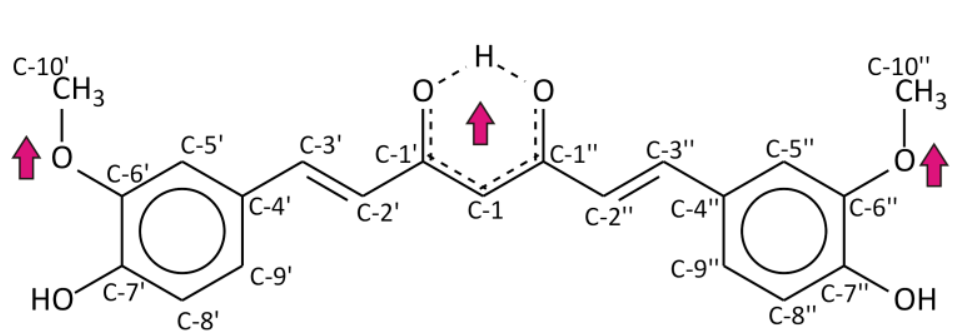

BINMEQ06 - BINMEQ08
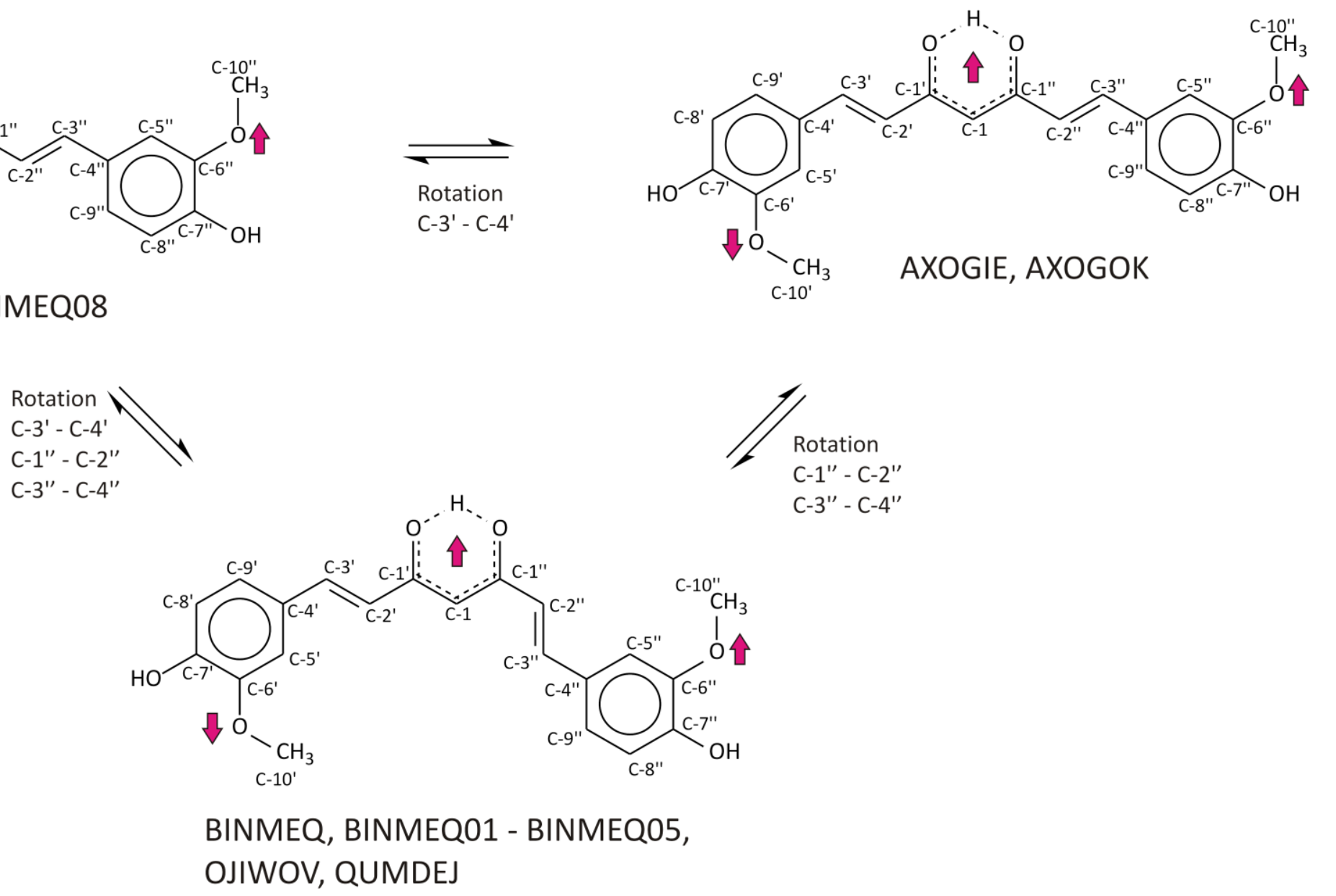

C-1" - C-2"

C-1" - C-2"

C-3" - C-4

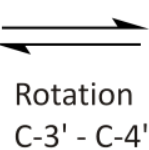

Rotation

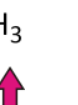


Figure 4: Curcumin with arrowed NOE contacts between ${ }^{1} \mathrm{H}$ nuclei and average ${ }^{1} \mathrm{H}-{ }^{1} \mathrm{H}$ internuclear distances $(\AA)$. $2.50 \AA$ is the reference distance.

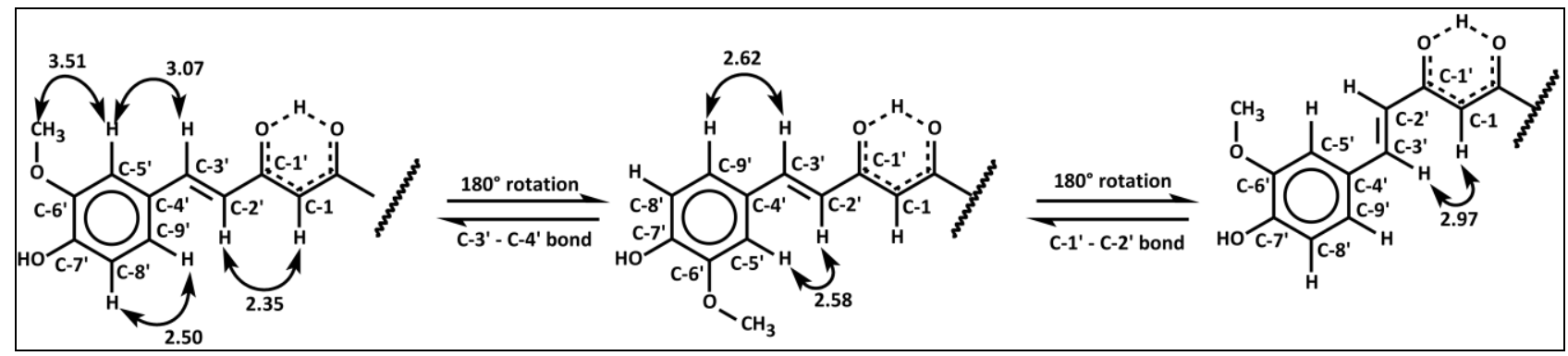

Figure S5: Curcumin with arrowed NOE contacts between ${ }^{1} \mathrm{H}$ nuclei and average ${ }^{1} \mathrm{H}-{ }^{1} \mathrm{H}$ internuclear distances $(\AA ̊)$. $2.50 \AA$ is the reference distance.

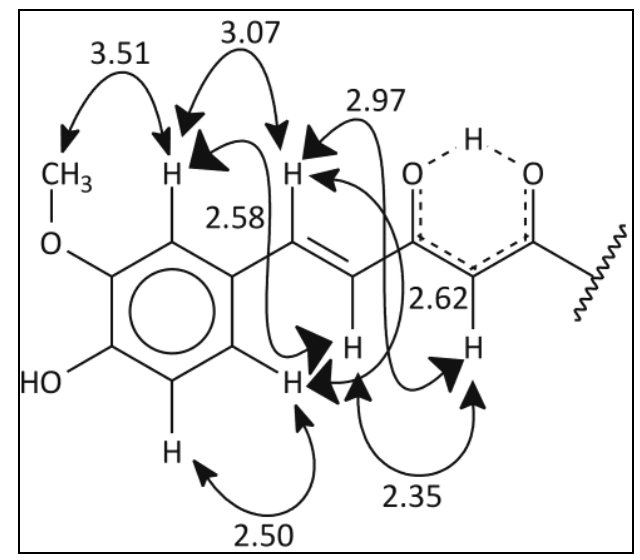

Figure 5: NAMFIS-selected conformations for curcumin in DMSO.

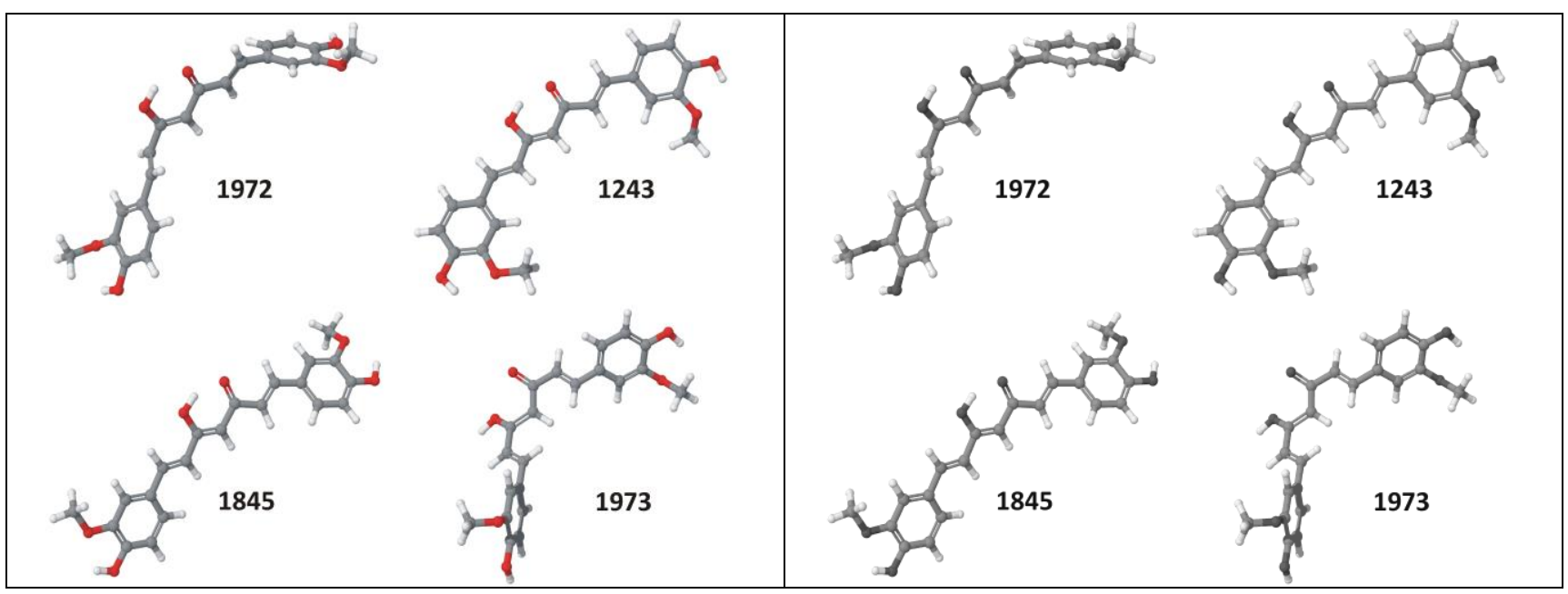


Figure 5: NAMFIS-selected conformations for curcumin in DMSO.
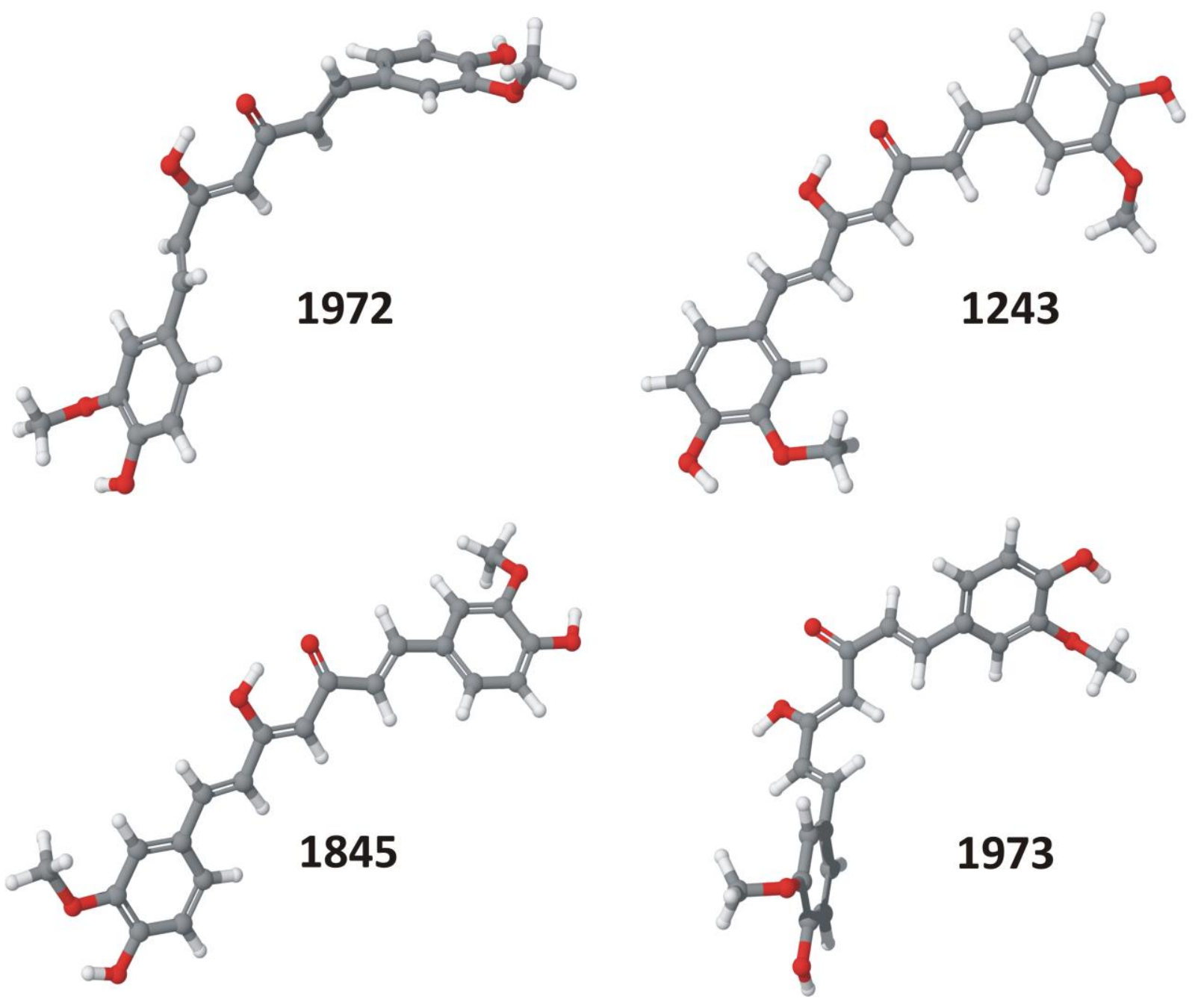
Figure 6: NAMFIS-1845 (green) and BINMEQ06 (RMSD $1.09 \AA ̊$ ).

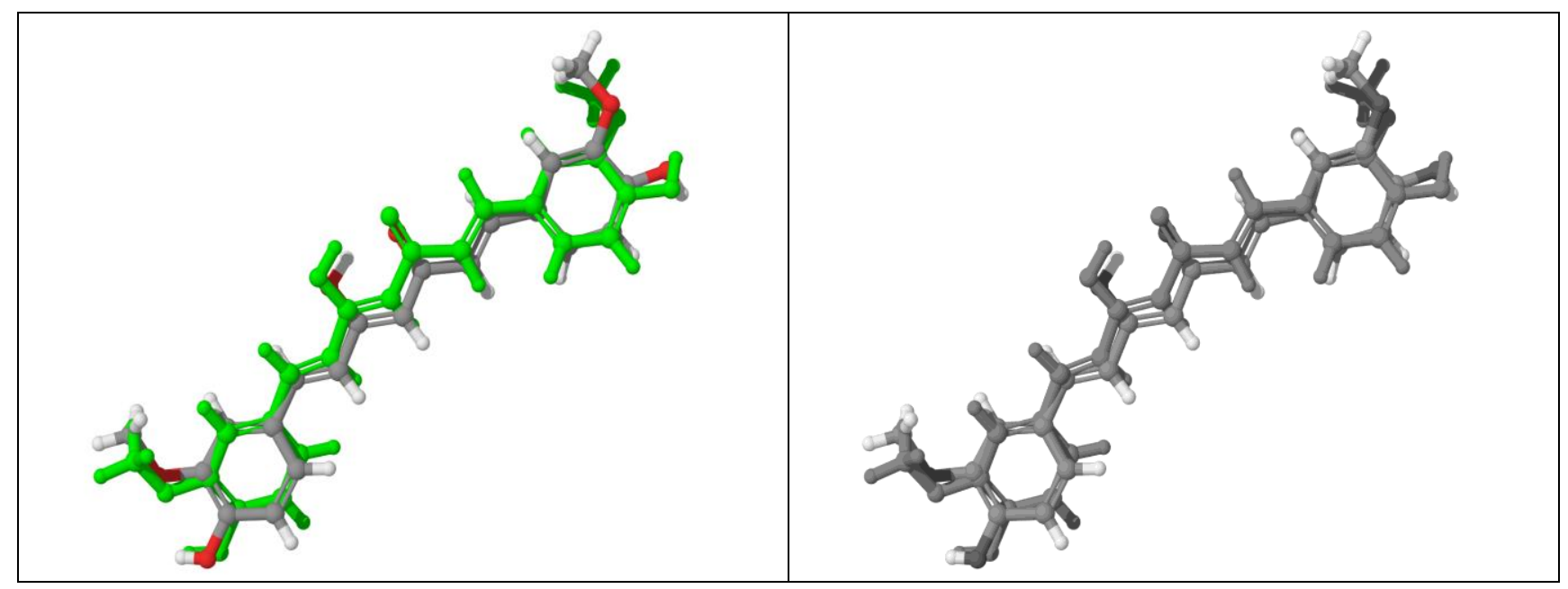

Figure 7: NAMFIS-1243 (blue) and BINMEQ with single-bond rotation about C-1" - C-2" (RMSD $2.47 \AA$ Å).

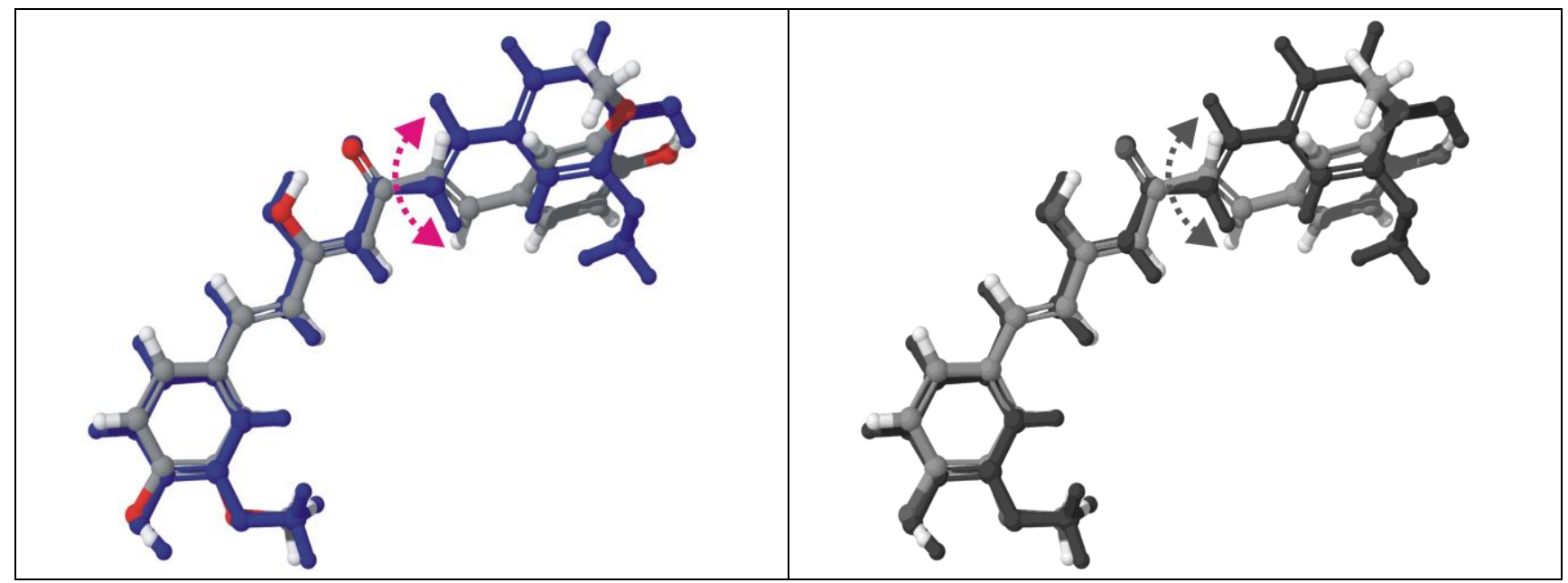

Figure 58: NAMFIS-1243 (blue) and BINMEQ04 with single-bond rotation about C-1' - C-2' (RMSD $1.97 \AA$ A).

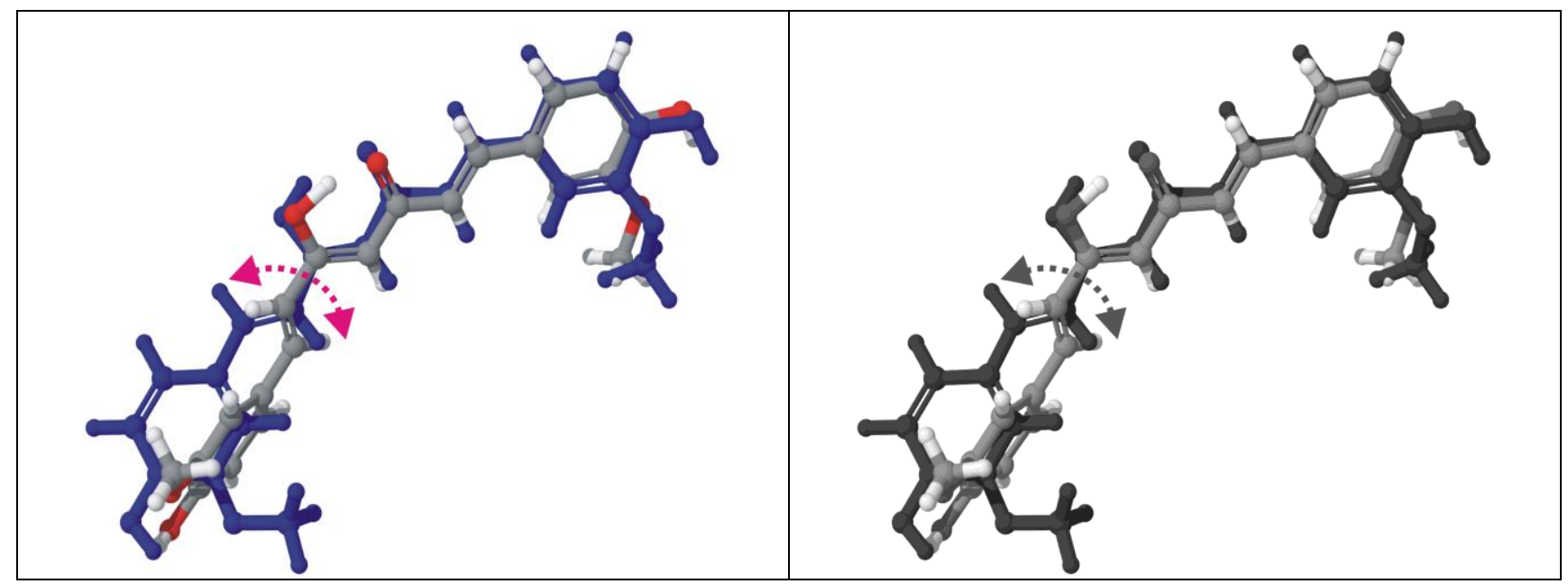

